\title{
Laser induced protonation of free base porphyrin in chloroform results in the enhancement of positive nonlinear absorption due to conformational distortion
}

\author{
Monika Zawadzka, ${ }^{a}$ Jun $\mathrm{Wang}^{b}$, Werner J. Blau ${ }^{c}$ and Mathias O. Senge ${ }^{{ }^{*} a}$ \\ ${ }^{a}$ School of Chemistry, SFI Tetrapyrrole Laboratory, Trinity Biomedical Sciences Institute, 152-160 Pearse Street, \\ Trinity College Dublin, Dublin 2, Ireland \\ ${ }^{b}$ Key Laboratory of Materials for High Power Lasers, Shanghai Institute of Optics and Fine Mechanics, Chinese \\ Academy of Science, 201800, Shanghai, China \\ ${ }^{c}$ School of Physics, Trinity College Dublin, Dublin 2, Ireland
}

Received date (to be automatically inserted after your manuscript is submitted)

Accepted date (to be automatically inserted after your manuscript is accepted)

\begin{abstract}
Free base porphyrin in chloroform solution irradiated with focused laser beam, in an open Z-scan set-up, changed color from purple to green, which was associated with protonation of the porphyrin with protonic species from photodegradation of the solvent. Protonation is demonstrated as a simple means to improve the optical limiting performance of free base porphyrin nonlinear optical (NLO) materials.
\end{abstract}

KEYWORDS: porphyrins, nonlinear optics, optical limiters, tetrapyrroles, conformational analysis

*Correspondence to: Mathias O. Senge, e-mail: sengem@tcd.ie, phone: +353-1-896-8537, fax: +353-1-896-8536 


\section{INTRODUCTION}

Nitrogen atoms in the central core of the porphyrin can be protonated under acidic conditions to form porphyrin diacids resulting in conformational changes leading to highly nonplanar structures [1,2]. The conformational changes are manifested in variations of the photophysical properties. The ground state absorption bands are shifted bathochromically and the number of Q-bands is reduced upon protonation. Fluorescence, intersystem crossing and first excited singlet state lifetimes decrease upon protonation, as does the quantum yield for triplet state formation. Moreover, the ratio of the excited to ground state absorption cross-section increases upon protonation [3,4-7]. The latter change is especially desirable for application in passive optical limiting (OL) devices [8,9].

Protonation of free base porphyrins can be carried out deliberately simply by the adjustment of the $\mathrm{pH}$ of the solution. Recent reports indicate that protonation can also be achieved unintentionally by subjecting free base porphyrins to sonification in a variety of chlorinated solvents. The phenomenon was associated with the formation of acidic products from sonolysis of the solvents, which reacted with free-base porphyrin to give its protonated form [10]. Here we report a similar phenomena arising upon subjecting free base porphyrin in chloroform solution to a focused $532 \mathrm{~nm} 6 \mathrm{~ns}$ pulsed laser beam. We associate the phenomenon with the photodegradation of the solvent providing acidic species which transform the free base compound to the respective protonated form.

\section{EXPERIMENTAL}

Characterization of the nonlinear absorption (NLA) was carried out with open aperture Z-scan technique [10] with a $6 \mathrm{~ns}$ pulsed laser at $532 \mathrm{~nm}$ in HPLC grade chloroform and spectroscopic grade toluene. Briefly, the open Z-scan technique measures total transmittance through the sample as the function of incident laser intensity while the sample gradually moves along a focused Gaussian beam. Details concerning experimental set-up can be found in our previous publication [11]. For each of the samples several Z-scan experiments were performed. For each subsequent experiment the input energy of the laser was increased. A $1 \mathrm{~mm}$ cell length was used in the open Z-scan measurements and concentrations used in this study were in the range of $2-3 \times 10^{-4} \mathrm{M}$. The ground state absorption studies were carried out using a Shimadzu multiSpec-1505 instrument. 5,10-Di( $p$-tolyl)porphyrin $\mathbf{1}$ and 5,10-dibromo-15,20-di( $p$ tolyl)porphyrin 2 were synthesized according to published procedures [12,13]. Protonation of free base compounds was achieved upon addition of a few drops of trifluoroacetic acid (TFA). Likewise, a few drops of triethylamine (TEA) were added to prevent protonation in chloroform solution in additional experiment.

\section{RESULTS AND DISCUSSION}

Deliberate protonation of chloroform solution of compound $\mathbf{1}$ and $\mathbf{2}$ with TFA induced changes to the ground state absorption spectra. The number of Q-bands was reduced and the spectrum was shifted bathochromically. Addition of TFA resulted in a color change from purple to green (Fig. 1). Similar red shifts of the Soret and Q-bands observed upon protonation were reported before for porphyrins and were associated with the induction of a nonplanar deformation [13,14-16]. Reduction in the number of Q-bands is typically observed upon protonation or metal insertion and arises from an increase in the symmetry of the molecule. Note, that the absorption spectrum of compound 2, both in free-base and protonated form, is red-shifted in comparison to $\mathbf{1}$. This is in accordance with previous reports which showed that bromination of meso positions induces a bathochromic shift in the UV-vis absorption spectra [14,17].

$<$ Formula $1>$

$<$ Figure 1> 
We observed that a chloroform solution of 1 subjected to a focused Gaussian beam in an open Z-scan [10] experiment changed color from purple to green in the area where the laser penetrated the sample. The phenomenon was enhanced when the laser energy was increased further in subsequent experiments. The sample was shaken after each of experiment in order to refresh the experimental volume. No such phenomenon was observed by us before for free base porphyrins in other solvents such as toluene or DMF. We consider the photoinduced protonation of free base porphyrin with chloroform acting as a proton source as a possible explanation for the observed process. Photolysis of chlorinated solvents has been widely noted in the literature and has been demonstrated to provide different species including protons through various channels, depending on the solvent nature and experimental conditions used [18,19]. Further studies will be needed to explore and unravel the exact mechanism of the observed phenomenon.

In order to prove that protonation was indeed involved we carried out open Z-scan studies on a sample containing TEA in order to suppress protonation. Additionally, we investigated NLA of a fully protonated sample in chloroform with TFA. The formation of the green cloud was indeed inhibited in the sample, with TEA, however, only in experiments at lower input energies $(\sim \mathrm{E}<100 \mu \mathrm{J})$. When the energy was increased a green area close to the laser spot became apparent and it slowly disappeared after each experiment. This effect was less pronounced than in neat chloroform. The open Z-scan data were manipulated and plotted as normalized transmission versus pulse energy density and are shown in Fig. 2. We also present data recorded in toluene for comparison, which we reported before [20].

$<$ Figure $2>$

The three different samples investigated (chloroform solution of compound $\mathbf{1}$ and same solutions with added TEA or TFA) provided different NLA responses. The transmission dropped to the lowest minimal value for the fully protonated sample while the sample with added TEA exhibited the weakest drop in transmission. The curve representing studies in pure chloroform exhibited a pattern in between the other two curves. Fig. 2 shows that studies carried out in toluene, in which no color change was observed upon illumination, recovered those recorded for chloroform with added TEA over a broad fluence range. It should be noted that studies at very high input energies were inaccessible in toluene as the asymmetric profile of Z-scan traces indicated the onset of degenerative processes. Based on these results it can be concluded that porphyrin in its protonated form absorbs light efficiently and, secondly, that addition of base inhibits the process leading to an enhancement of the OL performance in pure chloroform porphyrin solution. As protonated species are highly absorptive to laser light, their formation results in an improvement of the OL efficiency which we believe explains the response recorded for neat chloroform solutions of $\mathbf{1}$. As a color change was observed for studies at higher input energies for the sample with added TEA, protonation cannot be ruled out fully for these experiments. However, as the NLA curve representing studies in chloroform with added TEA recovered the curve representing

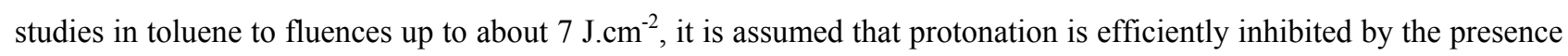
of TEA up to that fluence. It can be observed that two curves start to diverge in the higher fluence regime. However, it is difficult to assess if this arises due to laser induced protonation or is associated with solvent effects akin to those recently demonstrated by us to impact the NLA response of compound 1 and other $5,10-\mathrm{A}_{2} \mathrm{~B}_{2}$ porphyrins [20]. The curve representing studies in chloroform diverged from the one of the sample with added TEA which inhibited protonation at very low input fluences. Thus, it can be concluded that fluences on the order of $0.3 \mathrm{~J} . \mathrm{cm}^{-2}$ are sufficient to trigger the effective formation of protonated species of compound $\mathbf{1}$.

Recently, we demonstrated that compound 1 in its free base form (in toluene and DMF) and other 5,10- $A_{2} B_{2}$ porphyrins, exhibit a complex NLA behavior which can be rationalized with a four-level model. The model assumed consecutive one- and two-photon excited state absorption [20]. This four-level model was successfully used to fit the responses recorded in chloroform. An illustration of the model and the definition of the parameters used in NLA 
response fitting are given in Fig. 3. The best fit parameters used in the modeling of the responses are listed in Table 1. Results obtained for studies on compound 1 in toluene can be found elsewhere [20].

$<$ Figure $3>$

$<$ Table 1>

The theoretical curves diverged from the experimental data in the higher fluence regime for studies carried out in neat chloroform and solutions with added TEA. Most likely, the concentration of the protonated species varies with the input fluence within the experimental volume giving rise to the observed deviations. More complex procedures of data fitting, which would have to consider concentration variations of different species with the input fluence, possibly could provide a better fit. The theoretical curve matched well the experimental data for the fully protonated compound with TFA over the whole fluence range investigated. The applicability of the model implies that protonation does not affect the excited state structure of compound 1.

It can be observed that $\kappa_{1}$ and $\kappa_{2}$, being the ratios of the excited one- and two-photon absorption- to ground state absorption cross-section, and the saturation intensities $I_{1}$ and $I_{2}$, increase for the studies carried out on the three solutions in the following order: chloroform solution with added TEA < neat chloroform solution $<$ chloroform solution with added TFA. Meanwhile, $I_{3}$ decreases in the same order (Table 1). We associate the stronger transmission drop observed for the following three samples with an increase in parameters $\kappa_{l}$ and $\kappa_{2}$. The finding that protonated species exhibit a higher ratio of the excited to ground state absorption and better OL efficiency agrees with previous reports. According to our previous studies on the effect of the particular parameters on NLA performance, the trends observed for saturation intensities $I_{1}, I_{2}$ and $I_{3}$ weaken the OL performance of the protonated sample. However, clearly this effect is negligible in reference to that exerted by high $\kappa_{1}$ and $\kappa_{2}$ obtained for protonated porphyrin. Higher $I_{l}$ causes the saturated regime of one-photon excited state absorption to be accessed more slowly with input fluence. A similar conclusion refers to high $I_{2}$ in reference to two-photon processes. Provided that $\kappa_{2}$ is high enough in reference to $\kappa_{l}$, which was shown to be the case in the present studies, high $I_{2}$ weakens the transmission drop. This happens as a more effective two-photon process is suppressed. As far as $I_{3}$ is concerned, we found previously that high $I_{3}$ is important to prevent consecutive processes arising from state $N_{3}$. As these processes are highly unabsorptive their contribution lowers the transmission drop which consequently leads to RSA/SA turnover [20].

As protonation turned out to be an efficient means of improving the OL performance, we decided to investigate its effect on NLA of another compound, i.e. porphyrin 2. Replacement of the hydrogen atoms at two meso positions with bromine in $\mathbf{1}$ gave compound 2. The NLA response of compound $\mathbf{2}$ in its protonated form (with TFA) was studied in toluene. For comparison, data of the free base form in toluene are included [20]. Additionally, we also carried out open Z-scan studies on compound 2 in neat chloroform solution (Fig. 4).

$<$ Figure $4>$

Compound 2 is characterized by high linear absorption at the experimental wavelength of $532 \mathrm{~nm}$ which interrogated the compound's Q band. Provided that the absorption arising from the excited state is higher than that arising from the ground state the material exhibits SA. Such a behavior, although weakly pronounced, was detected for compound 2 in

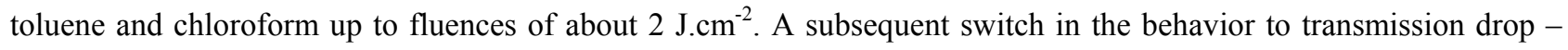
RSA - could be observed for both solvents at higher fluences. Another switch in behavior, again to SA, was observed for studies in toluene at even higher fluences. Again, this NLA behavior could be modeled using our four-level model [20]. The experimental wavelength of $532 \mathrm{~nm}$ was no longer in the Q-band wavelength range for the protonated form of compound 2 (Fig. 1). The fully protonated compound exhibited RSA/weak SA (Fig. 4). The responses recorded in chloroform recovered that in toluene over a broad fluence range up to $3.5 \mathrm{~J}_{\mathrm{cm}} \mathrm{cm}^{-2}$. This implies that compound 2 has a much weaker affinity to protonation than compound 1. Clearly, effective protonation would lead to a strong transmission drop as was observed for the dication of compound 2. It remains open whether the observed switch from 
SA to RSA in chloroform is associated with induced protonation or a solvent effect, which was previously demonstrated to shift SA/RSA turnover fluence [21]. Nevertheless, different parameters follow similar changes upon protonation as those observed for compound 1. $\kappa_{1}, \kappa_{2}, I_{1}$ and $I_{2}$ increased and $I_{3}$ decreased for a fully protonated sample in reference to the free base form. Additionally, it was observed that the fully protonated compound exhibited RSA/SA turnover at lower fluence that the free base compound which we associate with higher $I_{3}$ obtained for the latter form of the compound.

\section{CONCLUSIONS}

Protonation of free base porphyrins is a quick and easy way of enhancing their optical limiting efficiency. Special care must be taken while performing studies in chloroform solutions on free base porphyrins with focused laser beams as compounds can undergo protonation under the experimental conditions, which affects their photophysical properties.

\section{ACKNOWLEDGEMENTS}

This work was supported by a grant from Science Foundation Ireland (SFI P.I. 09/IN.1/B2650). J.W. received financial support from the 100-Talent Program of the Chinese Academy of Sciences, the National Natural Science Foundation of China (NSFC, Grant No. 61178007), and the Science and Technology Commission of Shanghai Municipality (STCSM Nano Project, Grant No. 11nm0502400).

\section{REFERENCES}

1. Stone A, Fleischer EB. J. Am. Chem. Soc. 1968; 90: 2735-2748.

2. Senge MO, Forsyth TP, Nguyen LT, Smith KM. Angew. Chem., Int. Ed. 1995; 33: 2485-2487.

3. Chirvony VS, van Hoek A, Galievsky VA, Sazanovich IV, Schaafsma TJ, Holten D. J. Phys. Chem. B 2000; 104: 9909-9917.

4. Liu ZB, Zhu Y, Zhu YZ, Tian JG, Zheng JY. J. Phys. Chem. B 2007; 111: 14136-14142.

5. Goncalves PJ, Borissevitch IE, Zilio SC. Chem. Phys. Lett. 2009; 469: 270-273.

6. Gupta I, Ravikanth M. J. Photochem. Photobiol., A 2006; 177: 156-163.

7. Goncalves PJ, De Boni L, Neto NMB, Rodrigues JJ, Zilio SC, Borissevitch IE. Chem. Phys. Lett. 2005; 407: 236241.

8. Sheikbahae M, Said AA, Wei TH, Hagan DJ, Vanstryland EW. IEEE J. Quantum Electron. 1990; 26: 760-769.

9. Senge MO, Fazekas M, Notaras EGA, Blau WJ, Zawadzka M, Locos OB, Mhuircheartaigh EMN. Adv. Mater. 2007; 19: 2737-2774.

10. Mhuircheartaigh EMN, Blau WJ, Prato M, Giordani S. Carbon 2007; 45: 2665-2671.

11. Zawadzka M, Wang J, Blau WJ, Senge MO. Chem. Phys. Lett. 2009; 477: 330-335.

12. Hatscher S, Senge MO. Tetrahedron Lett. 2003: 44: 157-160.

13. Senge MO, Ryppa C, Fazekas M, Zawadzka M, Dahms K. Chem. Eur. J. 2011; 17: 13562-13573.

14. Liu ZB, Zhu Y, Zhu YZ, Tian JG, Zheng YZ. J. Phys. Chem. B 2007; 111: 14136-14142.

15. Senge MO, Kalisch WW. Z. Naturforsch. 1999; 54b: 943-959.

16. Senge MO. Z. Naturforsch. 2000; 55b: 336-344.

17. Bonnett R, Harriman A, Kozyrev AN. J. Chem. Soc., Faraday Trans. 1992; 88: 763-769.

18. Poveda JC, Guerrero A, Alvarez I, Cisneros C. J. Photochem. Photobiol. A 2011; 220: 31-38. 
19. Bhardwaj VR, Rajgara FA, Vijayalakshmi K, Kumarappan V, Mathur D, Sinha AK. Phys. Rev. A 1998; 58: 38493855 .

20. Zawadzka M, Wang J, Blau WJ, Senge MO. J. Phys. Chem. A 2013; 117: 15-26.

21. Notaras EGA, Fazekas M, Doyle JJ, Blau WJ, Senge MO. Chem. Commun. 2007; 2166-2168. 


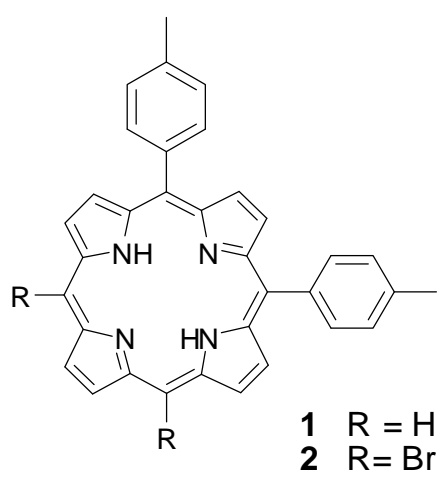

Formula 1

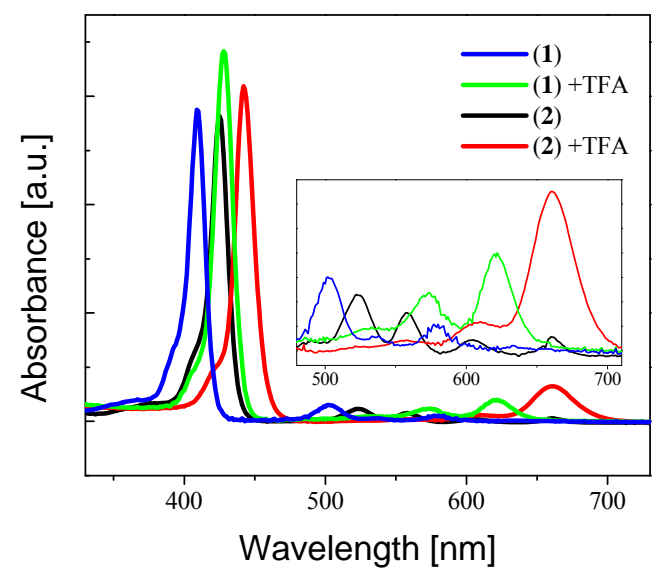

Figure 1. UV-vis spectra of compound $\mathbf{1}$ and $\mathbf{2}$ in free base and protonated forms (+TFA).

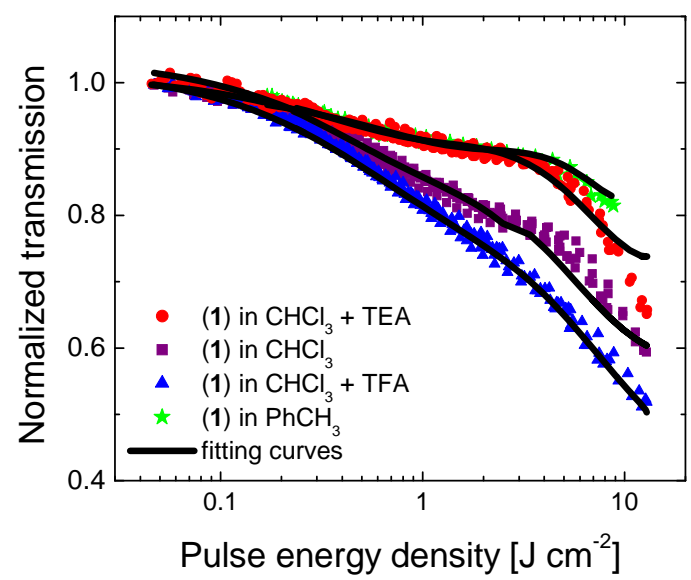

Figure 2. Optical limiting curves plotted as normalized transmission versus pulse energy density for compound 1 under various conditions. 




Figure 3. Illustration of the four-level model used to fit NLA responses along with the definition of the parameters $I_{1}, I_{2}, I_{3}, \kappa_{I}$ and $\kappa_{2}$. $\tau_{i}$ stands for the lifetime of the i-th state, $\sigma_{i}$ for the absorption cross-sections associated with i-th state. $I_{i}$ are saturation intensities associated with ground ( $(i=1)$ and excited states $(i=2,3), \kappa_{i}$ stands for the ratio of the excited to ground stated absorption crosssections.

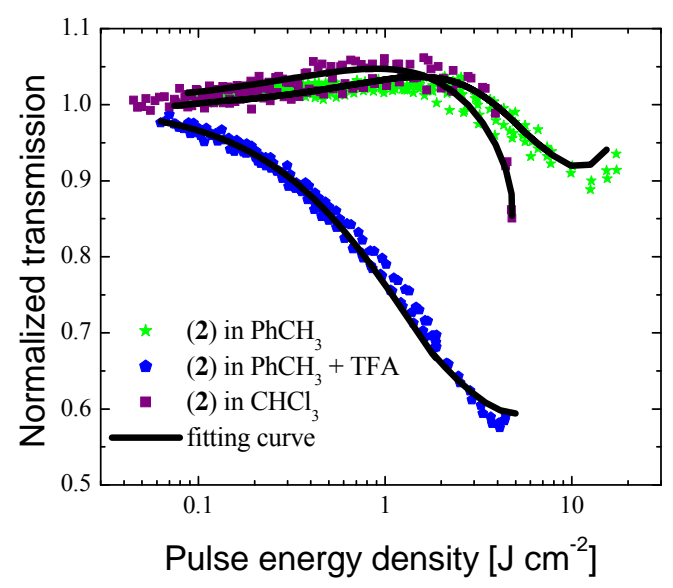

Figure 4. Optical limiting curves plotted as normalized transmission versus pulse energy density for compound 2 under different experimental conditions.

Table 1. Comparison of the best fit parameters used in the modeling of the responses of compound $\mathbf{1}$ and $\mathbf{2}$ (c $-\mathrm{chloroform}, \mathrm{t}-$ toluene). $\alpha_{0}$ is linear absorption coefficient at the experimental wavelength of $532 \mathrm{~nm}$.

\begin{tabular}{|c|c|c|c|c|c|c|}
\hline $\begin{array}{l}\text { \#l } \\
\text { solvent }\end{array}$ & $\begin{array}{l}\alpha_{0} \\
{\left[\mathrm{~cm}^{-1}\right]}\end{array}$ & $\kappa_{l}$ & $\begin{array}{l}\kappa_{2} \\
{\left[\mathrm{~cm}^{2} G W^{1}\right]}\end{array}$ & $\begin{array}{l}I_{l} \\
{\left[\mathrm{GWCm}^{-2}\right]}\end{array}$ & $\begin{array}{l}I_{2} \\
{\left[\mathrm{GWcm}^{-2}\right]}\end{array}$ & $\begin{array}{l}I_{3} \\
{\left[G W^{2} \mathrm{~cm}^{-4}\right]}\end{array}$ \\
\hline $1 / \mathrm{c}+\mathrm{TEA}$ & 1.31 & 3.1 & 3.6 & 0.14 & 0.35 & 3.8 \\
\hline $1 / \mathrm{c}$ & 1.34 & 4.5 & 5.8 & 0.20 & 0.45 & 3.5 \\
\hline $1 / \mathrm{c}+\mathrm{TFA}$ & 1.49 & 4.9 & 8.0 & 0.25 & 0.60 & 2.5 \\
\hline $2 / \mathrm{c}$ & 2.77 & 0.85 & 4.5 & 0.03 & 0.60 & 1.8 \\
\hline $2 / \mathrm{t}+\mathrm{TFA}$ & 1.08 & 12 & 8.0 & 0.53 & 1.00 & 1.1 \\
\hline
\end{tabular}

